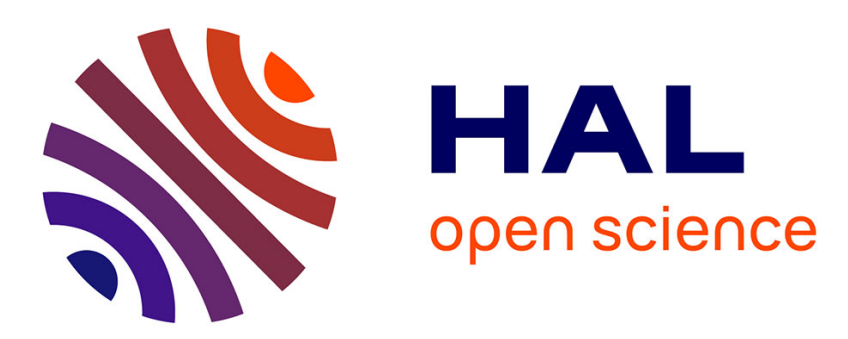

\title{
A Unifying Framework for Spatial and Temporal Diffusion in Diffusion MRI
}

Rutger H.J. Fick, Demian Wassermann, Marco Pizzolato, Rachid Deriche

\section{To cite this version:}

Rutger H.J. Fick, Demian Wassermann, Marco Pizzolato, Rachid Deriche. A Unifying Framework for Spatial and Temporal Diffusion in Diffusion MRI. Information Processing in Medical Imaging (IPMI), Jun 2015, Sabhal Mor Ostaig, Isle of Skye, United Kingdom. hal-01140070

\section{HAL Id: hal-01140070 \\ https://hal.inria.fr/hal-01140070}

Submitted on 7 Apr 2015

HAL is a multi-disciplinary open access archive for the deposit and dissemination of scientific research documents, whether they are published or not. The documents may come from teaching and research institutions in France or abroad, or from public or private research centers.
L'archive ouverte pluridisciplinaire HAL, est destinée au dépôt et à la diffusion de documents scientifiques de niveau recherche, publiés ou non, émanant des établissements d'enseignement et de recherche français ou étrangers, des laboratoires publics ou privés. 


\title{
A Unifying Framework for Spatial and Temporal Diffusion in Diffusion MRI
}

\author{
Rutger Fick ${ }^{\star}$, Demian Wassermann ${ }^{\star}$, Marco Pizzolato, and Rachid Deriche \\ Athena Project-Team, Inria Sophia Antipolis - Méditerranée, France ${ }^{\star \star}$
}

\begin{abstract}
We propose a novel framework to simultaneously represent the diffusion-weighted MRI (dMRI) signal over diffusion times, gradient strengths and gradient directions. Current frameworks such as the 3D Simple Harmonic Oscillator Reconstruction and Estimation basis (3DSHORE) only represent the signal over the spatial domain, leaving the temporal dependency as a fixed parameter. However, microstructurefocused techniques such as Axcaliber and ActiveAx provide evidence of the importance of sampling the dMRI space over diffusion time. Up to now there exists no generalized framework that simultaneously models the dependence of the dMRI signal in space and time. We use a functional basis to fit the $3 \mathrm{D}+\mathrm{t}$ spatio-temporal dMRI signal, similarly to the 3D-SHORE basis in three dimensional 'q-space'. The lowest order term in this expansion contains an isotropic diffusion tensor that characterizes the Gaussian displacement distribution, multiplied by a negative exponential. We regularize the signal fitting by minimizing the norm of the analytic Laplacian of the basis, and validate our technique on synthetic data generated using the theoretical model proposed by Callaghan et al. We show that our method is robust to noise and can accurately describe the restricted spatio-temporal signal decay originating from tissue models such as cylindrical pores. From the fitting we can then estimate the axon radius distribution parameters along any direction using approaches similar to AxCaliber. We also apply our method on real data from an ActiveAx acquisition. Overall, our approach allows one to represent the complete $3 \mathrm{D}+\mathrm{t}$ dMRI signal, which should prove helpful in understanding normal and pathologic nervous tissue.
\end{abstract}

\section{Introduction}

One of the unsolved quests of diffusion-weighted imaging (DW-MRI) is the reconstruction of the complete four-dimensional ensemble average propagator (EAP) describing the diffusion process of water molecules over three-dimensional space and diffusion time $(3 \mathrm{D}+\mathrm{t})$ in biological tissues. To the best of our knowledge, most recent imaging techniques focus on reconstructing the three-dimensional (3D) EAP using a fixed diffusion time. However, methods like Axcaliber [1] show

\footnotetext{
^ Rutger Fick and Demian Wassermann contributed equally to this work.

** This work was partially supported by the MOSIFAH ANR (France) Grant. M. Pizzolato thanks Olea Medical and the PACA Regional council for support
} 
the added value of incorporating different diffusion times when estimating the axon diameter in white matter tissue. Thus, a $3 \mathrm{D}+\mathrm{t}$ representation of the EAP may provide means to infer diffusion contrasts sensitive to axon diameters and other tissue characteristics. To our knowledge, no such representation has been proposed. We therefore propose an analytic model that enables the reconstruction of the complete $3 \mathrm{D}+\mathrm{t}$ EAP.

To relate the observed diffusion signal to the underlying tissue microstructure, we need to understand how the diffusion signal is influenced by the tissue geometry and properties. Starting from the concept of a single particle moving by Brownian motion, the movements of this particle over time are obstructed by surrounding tissue structures such as cell walls. Then considering a large group (ensemble) of particles, the average propagation of these particles will, depending on the length of the diffusion time, be more or less restricted by surrounding tissues. This ensemble average propagator (EAP) is denoted as $P(\mathbf{R}, \tau)$ with $\mathbf{R}$ the real displacement vector and $\tau$ the diffusion time.

In DW-MRI the EAP is estimated by obtaining diffusion-weighted images (DWIs). A DWI is obtained by applying two sensitizing diffusion gradients of pulse length $\delta$ to the tissue, separated by separation time $\Delta$. The resulting signal is 'weighted' by the average particle movements in the direction of the applied gradient. When these gradients are considered infinitely short $(\delta \approx 0)$, the relation between the measured signal $S(\mathbf{q}, \tau)$ and the $\operatorname{EAP} P(\mathbf{r}, \tau)$ is given by an inverse Fourier transform (IFT) [2] as

$$
P(\mathbf{R}, \tau)=\int_{\mathbb{R}^{3}} E(\mathbf{q}, \tau) e^{-2 \pi i \mathbf{q} \cdot \mathbf{r}} d \mathbf{q} \quad \text { with } \quad \mathbf{q}=\frac{\gamma \delta \mathbf{G}}{2 \pi}
$$

where $E(\mathbf{q}, \tau)=S(\mathbf{q}, \tau) / S_{0}$ is the normalized signal attenuation measured at position $\mathbf{q}$, and $S_{0}$ is the baseline image acquired without diffusion sensitization $(q=0)$. We denote $\tau=(\Delta-\delta / 3), q=|\mathbf{q}|, \mathbf{q}=q \mathbf{u}$ and $\mathbf{R}=R \mathbf{r}$, where $\mathbf{u}$ and $\mathbf{r}$ are $3 \mathrm{D}$ unit vectors and $q, R \in \mathbb{R}^{+}$. The wave vector $\mathbf{q}$ on the right side of Eq. (1) is related to pulse length $\delta$, nuclear gyromagnetic ratio $\gamma$ and the applied diffusion gradient vector G. Furthermore, the clinically used b-value is related to $\mathrm{q}$ as $b=4 \pi^{2} q^{2} \tau$. In accordance with the Fourier theory, measuring $E(\mathbf{q}, \tau)$ at higher q makes one sensitive to more precise details in $P(\mathbf{R}, \tau)$, while measuring at longer $\tau$ makes the recovered EAP more specific to the white matter structure.

The relation between the EAP and white matter tissue is often modeled by representing different compartments as pores [10]. Examples of these are parallel cylinders for aligned axon bundles and spherical pores for cell bodies and astrocytes. Several techniques exist to infer the properties of these pores such as their orientation or radius. Of these techniques many sample the 3D diffusion signal exclusively in q-space with one preset diffusion time [3][4][5]. Among the most used methods is diffusion tensor imaging (DTI) [3]. However, DTI is limited by its assumption that the signal decay is purely Gaussian over $\mathbf{q}$ and purely exponential over $\tau$. These assumptions cannot account for in-vivo observed phenomena such as restriction, heterogeneity or anomalous diffusion. Approaches that overcome the Gaussian decay assumption over $\mathbf{q}$ include the 
use of functional bases to represent the 3D EAP [4][5]. These bases reconstruct the radial and angular properties of the EAP by fitting the signal to a linear combination of orthogonal basis functions $E(\mathbf{q})=\sum_{i} c_{i} \Xi_{i}(\mathbf{q})$ with $\mathbf{c}$ the fitted coefficients. In the case of [5], these basis functions are eigenfunctions of the Fourier transform, allowing for the directly reconstruction of the EAP as $P(\mathbf{R})=$ $\sum_{i} c_{i} \Psi(\mathbf{R})$, where $\Psi=\operatorname{IFT}(\Xi)$. However, these approaches are not designed to include multiple diffusion times, and therefore cannot accurately model the complete $3 \mathrm{D}+\mathrm{t}$ signal.

The 3D EAP can be related to the mean pore (axon) sizes, e.g. mean volume, diameter and cross-sectional area, by assuming the q-space signal was acquired at a long diffusion time. In this case the diffusing particles have fully explored the tissue structure and thus the shape of the EAP is indicative of the shape of the tissue. This concept was proven in 1D-NMR [7][8][9] and extended to 3D with the 3D Simple Harmonic Oscillator Reconstruction and Estimation (3DSHORE) and Mean Apparent Propagator (MAP)-MRI [5] basis. However, this long diffusion time requirement is hard to fulfill in practice as the scanner noise begins to dominate the signal at higher diffusion times.

In contrast, in $1 \mathrm{D}+\mathrm{t}$ space, Axcaliber [1] samples both over $q$ and $\tau$ to estimate axon radius distribution. This allows it to overcome the long diffusion time constraint. However, though a $3 \mathrm{D}$-Axcaliber was briefly proposed [6], it is essentially a $1 \mathrm{D}$ technique that needs to fit a parametric model to a signal that is sampled exactly perpendicular to the axon direction. While this limits its applicability in clinical settings, this method thickly underlines the importance of including $\tau$ in the estimation of axon diameter properties.

Our main contribution in this paper is the generalization of the $3 \mathrm{D}-\mathrm{SHORE}$ model to include diffusion times. Our new model allows us to obtain analytic representations of the complete $3 \mathrm{D}+\mathrm{t}$ diffusion space from sparse samples of the diffusion signal attenuation $E(\mathbf{q}, \tau)$. In other words, our representation simultaneously represents the $3 \mathrm{D}+\mathrm{t}$ signal and EAP for any interpolated diffusion time. This allows the time-dependent computation of the orientation distribution function (ODF) previously proposed scalar measures such as the return-to-origin probability (RTOP) and return-to-axis probability (RTAP) [5].

While our new $3 \mathrm{D}+\mathrm{t}$ framework opens the door to many new ideas, in this work we consider an initial application of this framework by implementing the Axcaliber model to be used in 3D. In our procedure we first fit our model to a sparsely sampled synthetic $3 \mathrm{D}+\mathrm{t}$ data set consisting of cylinders with Gamma distributed radii. We then sample an Axcaliber data set from the $3 \mathrm{D}+\mathrm{t}$ representation perpendicular to the cylinder direction and fit Axcaliber to the resampled data. We compare this method with a previously proposed version of 3D-Axcaliber [6] that uses the composite and hindered restricted model of diffusion (CHARMED) model to interpolate the data points in $3 \mathrm{D}+\mathrm{t}$ space.

All contributions from this paper are publicly available on the Diffusion Imaging in Python (DiPy) toolkit [20]. http://nipy.org/dipy/. 


\section{Theory}

We propose an appropriate basis with respect to the dMRI signal by studying its theoretical shape over diffusion time $\tau$. The effect of diffusion time on the dMRI signal for different pore shapes has been extensively studies by Callaghan et al. [10]. In general, the equations for restricted signals in planar, cylindrical and spherical compartments can be formulated as:

$$
E(q, \tau)=\sum_{k} \beta_{k} e^{-\alpha_{k} \tau} \cdot f_{k}(q)
$$

where $\alpha_{k}$ and $\beta_{k}$ depend on the order of the expansion. Here $f_{k}(q)$ is a function that depends on the expansion order and value of $q$. The exact formulations can be found in equations (9), (13) and (17) in [10]. As Eq. (2) shows, every expansion order is given as a product of two functions: A negative exponential on $\tau$ with some order dependent scaling and a function $f_{k}(q)$ depending only on $q$. Therefore, an appropriate basis to fit the signal described in Eq. (2) should be a similar product of an exponential basis over $\tau$ and another spatial basis over q. We provide the formulation of our basis in the next section.

\subsection{Specific Formulation of the $3 \mathrm{D}+\mathrm{t}$ Basis}

In accordance with the theoretical model presented in Section 2 we fit the $3 \mathrm{D}+\mathrm{t}$ space with a functional basis that is both separable and orthogonal over both $\mathbf{q}$ and $\tau$. For the temporal aspect of the signal we choose to use an exponential modulated by a Laguerre polynomial, which together form an orthogonal basis over $\tau$. Then, following the separability of the signal, we are free to choose any previously proposed spatial basis to complete our $3 \mathrm{D}+\mathrm{t}$ functional basis. We choose to use the well-known 3D-SHORE basis [5] as it robustly recovers both the radial and angular features from sparse measurements [11]. Our combined basis finally describes the $3 \mathrm{D}+\mathrm{t}$ diffusion signal as

$$
E(\mathbf{q}, \tau)=\sum_{\{j l m\}}^{N_{\max }} \sum_{o=0}^{O_{\max }} c_{j l m o} S_{j l m}(\mathbf{q}) T_{o}(\tau)
$$

where $T_{o}(\tau)$ is our temporal basis with basis order $o$ and $S_{j l m}(\mathbf{q})$ is the 3DSHORE basis with basis orders $j \mathrm{~lm}$. Here $N_{\max }$ and $O_{\max }$ are the maximum spatial and temporal order of the bases, which can be chosen independently. We formulate the bases themselves as

$$
\begin{aligned}
S_{j l m}\left(\mathbf{q}, u_{s}\right) & =\sqrt{4 \pi} i^{-l}\left(2 \pi^{2} u_{s}^{2} q^{2}\right)^{l / 2} e^{-2 \pi^{2} u_{s}^{2} q^{2}} L_{j-1}^{l+1 / 2}\left(4 \pi^{2} u_{s}^{2} q^{2}\right) Y_{l}^{m}(\mathbf{u}) \\
T_{o}\left(\tau, u_{t}\right) & =\exp \left(-u_{t} \tau / 2\right) L_{o}\left(u_{t} \tau\right)
\end{aligned}
$$

where $u_{s}$ and $u_{t}$ are the spatial and temporal scaling factors. Here $\mathbf{q}=q \mathbf{u}, L_{n}^{(\alpha)}$ is a generalized Laguerre polynomial and $Y_{l}^{m}$ is the real spherical harmonics basis [12]. Here $j, l$ and $m$ are the radial order, angular order and angular 
moment of the 3D-SHORE basis which are related as $2 j+l=N+2$ with $N \in\left\{0,2,4 \ldots N_{\max }\right\}[5]$.

Furthermore, we require data-dependent scaling factors $u_{s}$ and $u_{t}$ to efficiently fit the data. We calculate $u_{s}$ by fitting a tensor $e^{-2 \pi^{2} q^{2} u_{s}^{2}}$ to the signal values $E(\mathbf{q}, \cdot)$ for all measured $\mathbf{q}$. Similarly, we compute $u_{t}$ by fitting an exponential $e^{-u_{t} \tau}$ to $E(\cdot, \tau)$ for all measured $\tau$. Lastly, for a symmetric propagator in our $3 \mathrm{D}+\mathrm{t}$ basis (as is the case in $\mathrm{dMRI}$ ) we give the total number of estimated coefficients $N_{\text {coef }}$ as

$$
N_{\text {coef }}=\left(O_{\max }+1\right)\left(N_{\max } / 2+1\right)\left(N_{\max } / 2+2\right)\left(4 N_{\max } / 2+3\right) .
$$

For notation convenience, we use a linearized indexing of the basis functions in the rest of the paper. We denote $\Xi_{i}\left(\mathbf{q}, \tau, u_{s}, u_{t}\right)=S_{j l m(i)}\left(\mathbf{q}, u_{s}\right) T_{o(i)}\left(\tau, u_{t}\right)$ with $i \in\left\{1 \ldots N_{\text {coef }}\right\}$.

\subsection{Signal Fitting and Regularization}

As the measured signal always contains noise we need to regularize the coefficient estimation. Therefore, as our second contribution in this work, we provide the analytic form of the Laplacian regularization of our basis.

Following Eq. (3), we fit our basis using regularized least squares by first constructing a design matrix $\mathbf{Q} \in \mathbb{R}^{N_{\text {data }} \times N_{\text {coef }}}$ with $\mathbf{Q}_{i k}=\Xi_{k}\left(\mathbf{q}_{i}, \tau_{i}, u_{s}, u_{t}\right)$. We then fit the signal as

$$
\mathbf{c}=\operatorname{argmin}_{\mathbf{c}}\|\mathbf{y}-\mathbf{Q} \mathbf{c}\|^{2}+\lambda U(\mathbf{c})
$$

where $\mathbf{y}$ is the measured signal, $\mathbf{c}$ are the fitted coefficients and $\lambda$ is the weight for our Laplacian regularization $U(\mathbf{c})$. We define $U(\mathbf{c})$ as

$$
U(\mathbf{c})=\int_{\mathbb{R}}\left\|\nabla^{2} E_{\mathbf{c}}(\mathbf{q}, \tau)\right\|^{2} d \mathbf{q} d \tau
$$

with $\nabla^{2} E_{\mathbf{c}}(\mathbf{q}, \tau)=\sum_{i} c_{i} \nabla^{2} \Xi_{i}\left(\mathbf{q}, \tau, u_{s}, u_{t}\right)$ the Laplacian of the reconstructed signal. $U(\mathbf{c})$ can be rewritten in quadratic form as

$$
\mathbf{U}_{i k}=\int_{\mathbb{R}} \nabla^{2} \Xi_{i}\left(\mathbf{q}, \tau, u_{s}, u_{t}\right) \cdot \nabla^{2} \Xi_{k}\left(\mathbf{q}, \tau, u_{s}, u_{t}\right) d \mathbf{q} d \tau
$$

where the subscript $i k$ indicates the $i k^{t h}$ position in the regularization matrix. We use the orthogonality of the basis functions to compute the values of the regularization matrix to a closed form depending only on the basis orders and scale factors. For brevity here we give the formulation of $\mathbf{U}$ in the Appendix A. We finally estimate the coefficients using regularized least squares

$$
\mathbf{c}=\left(\mathbf{Q}^{\top} \mathbf{Q}+\lambda \mathbf{U}\right)^{-1} \mathbf{Q}^{\top} \mathbf{y} .
$$

We find the weight $\lambda$ through generalized cross-validation (GCV) [13]. We fit our model on both synthetic data generated using the theoretical signal model and real data. We describe the theoretical signal model in more detail in the next section. 


\subsection{Synthetic Data Generation and Axcaliber Model}

To validate our method we generate synthetic data using the Callaghan model [10]. In the case of a cylindrical (axonal) compartment this model simulates the restricted component perpendicular to the cylinder walls as:

$$
\begin{aligned}
E_{r}(q, \tau) & =\sum_{k} 4 \exp \left(-\beta_{0 k}^{2} D \tau / a^{2}\right) \times \frac{\left((2 \pi q a) J_{0}^{\prime}(2 \pi q a)\right)^{2}}{\left((2 \pi q a)^{2}-\beta_{0 k}^{2}\right)^{2}} \\
& +\sum_{n k} 8 \exp \left(-\beta_{n k}^{2} D \tau / a^{2}\right) \times \frac{\beta_{n k}^{2}}{\left(\beta_{n k}^{2}-n^{2}\right)} \times \frac{\left((2 \pi q a) J_{n}^{\prime}(2 \pi q a)\right)^{2}}{\left((2 \pi q a)^{2}-\beta_{n k}^{2}\right)^{2}}
\end{aligned}
$$

where $J_{n}^{\prime}$ are the derivatives of the $n^{t h}$-order Bessel function, $\beta_{n k}$ are the arguments that result in zero-crossings and the cylinders are of radius $a$. As Eq. (10) models diffusion for a single fiber population, this expression is extended as in Axcaliber to include contributions from a Gamma distribution of fiber diameters [1]. In fact, Eq. (10) is exactly the model that is fitted to the $1 \mathrm{D}+\mathrm{t}$ signal in Axcaliber. Following Eq. (3) in [17] we complete the model for a cylindrical compartment by adding a free diffusion component as

$$
E(\mathbf{q}, \tau)=E_{r}\left(q_{\perp}, \tau\right) \cdot E_{\text {free }}\left(q_{\|}, \tau\right) .
$$

where $q_{\|}=\langle\mathbf{q}, \mathbf{f}\rangle$ with $\langle\cdot\rangle$ the inner product and $\mathbf{f}$ the orientation of the cylinder. Using the free water diffusivity $D=3 \cdot 10^{-9} \mathrm{~m} / \mathrm{s}^{2}$, the parallel compartment is given as

$$
E_{\text {free }}\left(q_{\|}, \tau\right)=e^{-4 \pi^{2} \mathbf{q}^{2} D \tau} .
$$

\section{$3 \quad$ Experiments}

In this section we first validate our method using synthetic data generated using the theoretical Callaghan model [10]. We then apply our method on real data acquired for ActiveAx [15].

\subsection{Synthetic Data Experiments}

Using the theoretical model outlined in Section 2.3 we generate two axon populations with Gamma distributed radii. We choose the shape and scale parameters of the Gamma distribution similar to the optic nerve and sciatic nerve distributions presented in the Axcaliber paper [1]. We show the shapes of the Gamma distributions and corresponding restricted signal attenuations in Figure 1.

We sample Eq. (11) in q-points distributed according to [18]. For every diffusion time $\tau$ we sample different q-space shells at $q=\{0,2,5,10,30,50,70\} \mathrm{mm}^{-1}$. Each shell is sampled with $\{3,10,10,10,20,20,20\}$ samples, respectively. This acquisition is repeated for every diffusion time $\tau=\{10,20,40,60\}$ ms, leading to a total of 372 samples. We compute this data for both Gamma distributions for the signal fitting and Axcaliber experiments in the next sections. 


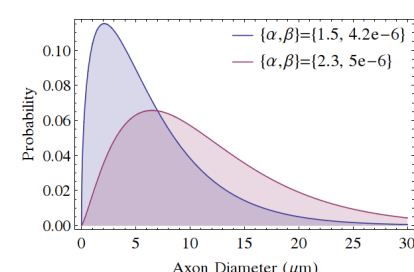

(a) Gamma Distributions

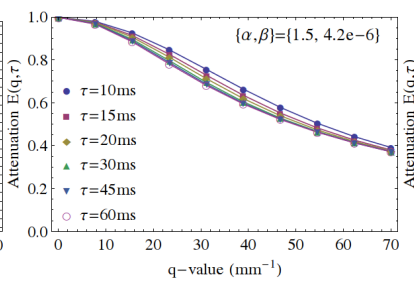

(b) Signal Optic Nerve

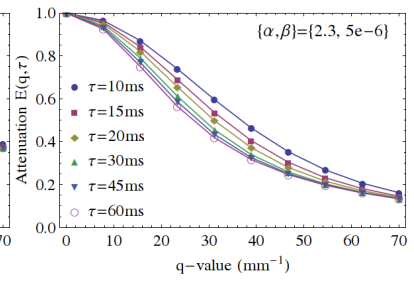

(c) Signal Sciatic Nerve

Fig. 1: Signals generated using the Callaghan model.

\subsection{Signal fitting and Effect of Regularization}

In our first experiment we test how many spatial or temporal basis functions we need to fit a $3 \mathrm{D}+\mathrm{t}$ diffusion signal. We choose to study in the case of restricted diffusion in a cylindrical compartment, since this is a good model for white matter tissue in highly organized areas. We generate the noiseless signal as described in Section 2.3 with the sampling scheme we described in Section 3.1. We then fit the signal with increasing maximum order for the spatial and temporal basis. We then compute the mean squared error (MSE) of the fitted signal compared to the ground truth. We show a heat map of the results in Figure 2a where we see that the signal fitting in this specific signal model only improves very little after a spatial order of 6 and a temporal order of 5. Using Eq. (5) this means we fit 300 coefficients to accurately represent the $3 \mathrm{D}+\mathrm{t}$ signal.

Using these settings for the maximum radial and temporal order we then study the effectiveness of our proposed Laplacian regularization when we (1) remove samples or (2) add noise to the data. In (1) we add a typical amount of noise to the data such that $\mathrm{SNR}=20$ and remove samples from the data in steps of 12 samples. We then compare the MSE of the fitted signal with the noiseless whole signal of 372 samples. We present the results in Figure 2b, where you can see that the regularized $3 \mathrm{D}+\mathrm{t}$ basis (in red) has significantly lower MSE than the unregularized basis. You can also see that the MSE error starts to increase when the number of samples is reduced below 300. In (2) we set the number of samples to 300 and increase the noise from $\mathrm{SNR}=5$ to $\mathrm{SNR}=50$. In Figure 2c you can again see that our regularized basis has lower MSE values.

\subsection{Three Dimensional Axcaliber from 3D+t}

With this experiment we explore an application of our $3 \mathrm{D}+\mathrm{t}$ basis by including Axcaliber [1]. Axcaliber is a method that can estimate the parameters of the Gamma distribution of the fiber radii by fitting the Gamma distributed version of Eq. (10) to the signal over both $\mathbf{q}$ and $\tau$. However, it requires that the data is sampled exactly perpendicular to the axon population, which makes it impractical for clinical use. 


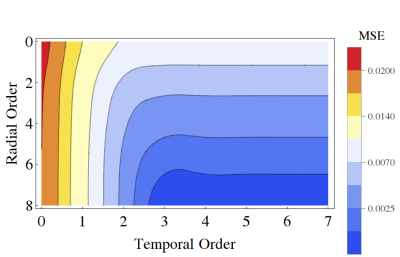

(a) Different Basis Orders

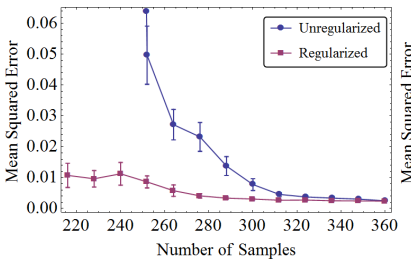

(b) Reducing Samples

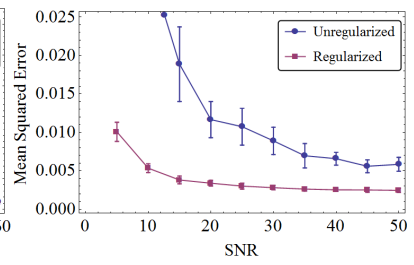

(c) Increasing Noise

Fig. 2: (a) A heat map representing the mean squared error (MSE) of the basis fitting for different maximum radial and temporal orders. (b) The effect of reducing the number of samples on the MSE. (c) The effect of increasing the noise in the data on the MSE.

An advantage of our model is that we can apply Axcaliber in any direction by first fitting the entire 3D+t signal with Eq. (9) and then sampling the data again perpendicular to the observed fiber direction. We compare our approach with a similar proposal [6] previously made using the composite and hindered restricted model of diffusion (CHARMED) model [16]. In contrast to our method, which assumes no a-priori shape on the EAP, the CHARMED model fits specific hindered and restricted compartments to the signal [17].

In this experiment we simulate 300 signal samples at $\mathrm{SNR}=20$ using Eq. (11) for both Gamma distributions presented in Figure 1. In this experiment we, without loss of generality, fix the axon direction along the z-axis and only consider the intra-axonal signal (i.e. no hindered compartment). We then fit our model with a radial order of 6 and temporal order of 5 . We fit CHARMED using 3 restricted compartments. Then, as the signal in a cylindrical compartment should be axially symmetric, we sample 10 different directions on the plane perpendicular to $\mathrm{z}$ and average the signals to reduce the effects of noise. The Axcaliber data set consists of $q=\{0,10,20,30,40,50,60,70\}$ at $\tau=\{10,20,30,40,50,60\}$, resulting in 42 samples. We repeat the experiment 100 times.

Figure 3 shows box plots of the recovered shape and scale parameters $\alpha$ and $\beta$ from the optic and sciatic nerve data sets for both our $3 \mathrm{D}+\mathrm{t}$ method and CHARMED. The blue box contains values that are within the first and third quartile of the obtained values, while the horizontal line in the middle is the median value. On the right we also show the estimated mean radius, which can be directly estimated from the gamma distribution as $\langle R\rangle=\alpha \beta$. The green line represents the ground truth. It can be seen that the ground truth is always within the first and third quartile for our method, while CHARMED typically overestimates $\beta$ and underestimates $\alpha$.

\subsection{Axon Diameter from Monkey Data}

As a real data experiment we apply our model to an ActiveAx data set [15][19] of an ex-vivo monkey brain. The data set consists of four shells with 93 samples 


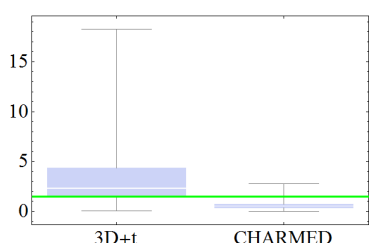

(a) $\alpha$ optic nerve

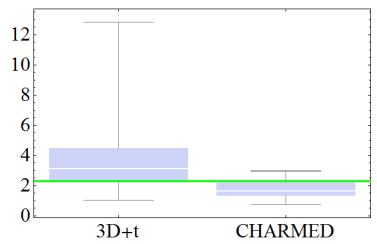

(d) $\alpha$ sciatic nerve

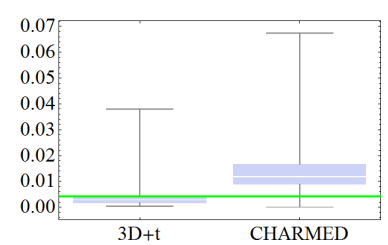

(b) $\beta$ optic nerve

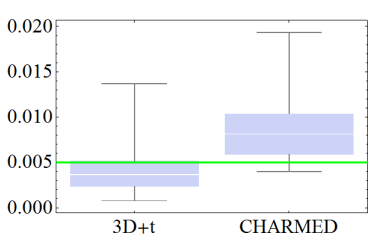

(e) $\beta$ sciatic nerve

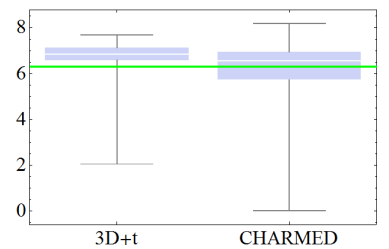

(c) $\langle R\rangle$ optic nerve $(\mu m)$

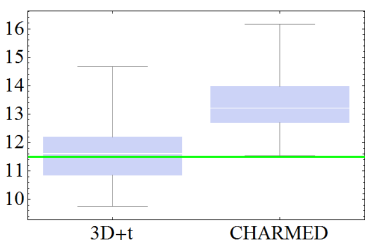

(f) $\langle R\rangle$ sciatic nerve $(\mu m)$

Fig. 3: The recovered shape $\alpha$, scale $\beta$ and average axon radius $\langle R\rangle$ for the optic nerve (top row) and sciatic nerve (bottom row) data sets. The green line is the ground truth.

each, and uses gradient strengths $G=\{.14, .14, .14, .13\} \mathrm{T} / \mathrm{m}$, separation times $\Delta=\{35.78,16.7,16.7,45.9\} \mathrm{ms}$ and pulse lengths $\delta=\{17.74,10.15,10.17,7.17\} \mathrm{ms}$, respectively. As you can see the pulse lengths $\delta$ are comparable to $\Delta$ and differ between acquisition shells, which makes it not ideal for our method. However, it is the only data set publicly available that has different measurements in $\Delta$.

We use the provided mask of the corpus callosum [15] and fit Eq. (9) to the data using a radial order of 6 and a temporal order of 3 . We then use the approach in Section 3.3 and compute the mean axon radii. We present these results in Figure 4 . We can see that, while the results are somewhat noisy, we find smaller radii near the splenium and genu (around 2-3 $\mu \mathrm{m}$ ) and bigger near the midbody (around 3-4 $\mu \mathrm{m}$ ). This trend roughly follows what was found in [15], showing that our method obtains reasonable results even in this data.
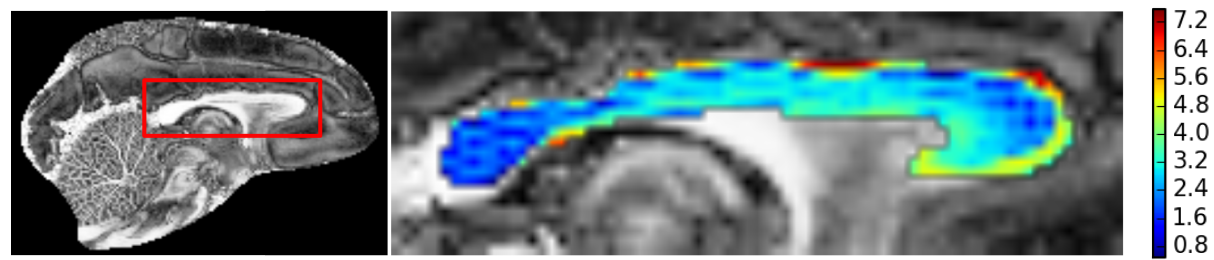

Fig. 4: A fractional anisotropy (FA) map of the ex-vivo monkey brain (left) and the estimated axon radii in the corpus callosum (right). 


\section{Discussion and Conclusions}

Our main contribution in this work is a novel framework to simultaneously represent the diffusion-weighted MRI (dMRI) signal over diffusion times, gradient strengths and gradient directions. Our framework is based on the theoretical model of restricted diffusion by Callaghan et al. [10] and uses an orthogonal functional basis to fit the spatio-temporal diffusion signal over q-space and diffusion times, which together we call $3 \mathrm{D}+\mathrm{t}$ space. To the best of our knowledge, we are the first to propose a method to represent the $3 \mathrm{D}+\mathrm{t}$ space using a functional basis. In accordance with the separability of our functional basis, we can choose our spatial and temporal basis independently. We proposed to fit the temporal signal using a basis of negative exponentials modulated by Laguerre polynomials, while we chose to fit the spatial signal using the 3D-SHORE basis. One theoretical limitation of this choice of basis is that it does not directly model free water diffusion. However, the free water diffusion signal with the parameters found in WM dMRI is well-represented by our basis, hence the theoretical limitation does not seem to represent a major issue in our dMRI applications. More importantly, this formulation retains all properties of the 3D-SHORE basis, but with the added information over diffusion time. These benefits include a timedependent analytic representation of the dMRI signal and diffusion propagator.

Our formulation also allows for the efficient regularization of the basis in the form of the minimization of the Laplacian. We provide the analytic solution of this Laplacian regularization depending only on the basis order and scaling factors, allowing for instant computation of the regularization matrix for any combination of basis order. We show on synthetic data that it effectively regularizes the basis fitting.

Furthermore, we explored a possible application of our 3D+t framework by including Axcaliber [1]. We showed on synthetic data that by first fitting our basis to a sparse $3 \mathrm{D}+\mathrm{t}$ sampling, we can accurately interpolate an Axcaliber data set along any direction. This allowed us to estimate the axon radius distribution parameters despite not sampling directly perpendicular to the axon orientation. We compared this approach with a similar proposal using CHARMED [16] and we showed that our approach is more appropriate to fit the $3 \mathrm{D}+\mathrm{t}$ signal.

In its current form our framework effectively represents the $3 \mathrm{D}+\mathrm{t}$ diffusion signal and allows us to freely interchange the spatial basis to any other basis that more readily fits anisotropic data. For instance, the MAP-MRI basis [5] could be used, which can also be extended to include the analytic Laplacian regularization. Therefore, the framework presented in this work is meant as an original and important step towards complete $3 \mathrm{D}+\mathrm{t}$ imaging in diffusion MRI, and provides great potential to better understand the diffusion signal in normal and pathologic nervous tissue.

\section{Appendix A: Analytic Laplacian Regularization}

Here we compute the analytic form of the Laplacian regularization matrix in Eq. (8). As our basis is separable in $\mathbf{q}$ and $\tau$, we can separate the Laplacian over our 
basis function $\Xi_{i}$ in a the spatial and temporal Laplacian as

$$
\nabla^{2} \Xi_{i}\left(\mathbf{q}, \tau, u_{s}, u_{t}\right)=\left(\nabla_{\mathbf{q}}^{2} S_{i}\left(\mathbf{q}, u_{s}\right)\right) T_{i}\left(\tau, u_{t}\right)+S_{i}\left(\mathbf{q}, u_{s}\right)\left(\nabla_{\tau}^{2} T_{i}\left(\tau, u_{t}\right)\right)
$$

with $\nabla_{\mathbf{q}}^{2}$ and $\nabla_{\tau}^{2}$ the Laplacian to either $\mathbf{q}$ or $\tau$. We then rewrite Eq. (8) as

$$
\begin{aligned}
\mathbf{U}_{i k} & =\overbrace{\int_{\mathbb{R}}\left(\nabla_{\mathbf{q}}^{2} S_{i}\right)\left(\nabla_{\mathbf{q}}^{2} S_{k}\right) d \mathbf{q} \int_{\mathbb{R}} T_{i} T_{k} d \tau}^{\mathbf{U}_{i k}^{\mathrm{I}}}+\overbrace{\int_{\mathbb{R}}\left(\nabla_{\mathbf{q}}^{2} S_{i}\right) S_{k} d \mathbf{q} \int_{\mathbb{R}} T_{i}\left(\nabla_{\tau}^{2} T_{k}\right) d \tau}^{\mathbf{U}_{i k}^{\mathrm{IIa}}} \\
& +\underbrace{\int_{\mathbb{R}} S_{i}\left(\nabla_{\mathbf{q}}^{2} S_{k}\right) d \mathbf{q} \int_{\mathbb{R}}\left(\nabla_{\tau}^{2} T_{i}\right) T_{k} d \tau}_{\mathbf{U}_{i k}^{\mathrm{IIb}}}+\underbrace{\int_{\mathbb{R}} S_{i} S_{k} d \mathbf{q} \int_{\mathbb{R}}\left(\nabla_{\tau}^{2} T_{i}\right)\left(\nabla_{\tau}^{2} T_{k}\right) d \tau}_{\mathbf{U}_{i k}^{\mathrm{III}}}
\end{aligned}
$$

where $\mathbf{U}_{i k}^{\mathrm{IIa}}=\mathbf{U}_{k i}^{\mathrm{IIb}}$. In all cases the integrals over $\mathbf{q}$ and $\tau$ can be calculated to a closed form using the orthogonality of the spherical harmonics, and Laguerre polynomials with respect to weighting function $e^{-x}$. The closed form of $\mathbf{U}^{\mathrm{I}}$ is

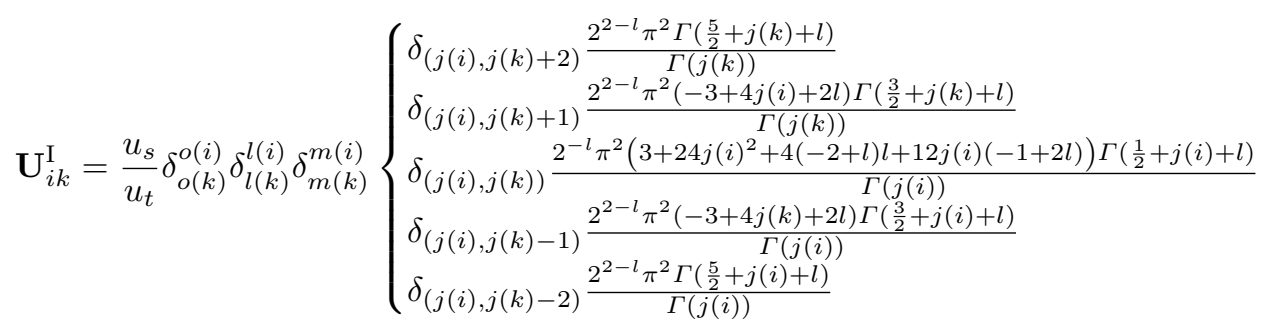

where $\delta$ is the Kronecker delta. Similarly computing $\mathbf{U}_{i k}^{\mathrm{II}}=\mathbf{U}_{i k}^{\mathrm{IIa}}+\mathbf{U}_{k i}^{\mathrm{IIb}}$ gives

$$
\begin{aligned}
\mathbf{U}_{i k}^{\mathrm{II}} & =\frac{u_{t}}{u_{s}} \delta_{l(k)}^{l(i)} \delta_{m(k)}^{m(i)}\left\{\begin{array}{l}
\delta_{(j(i), j(k)+1)} \frac{2^{-l} \Gamma\left(\frac{3}{2}+j(k)+l\right)}{\Gamma(j(k))} \\
\delta_{(j(i), j(k))} \frac{2^{-(l+1)}(1-4 j(i)-2 l) \Gamma\left(\frac{1}{2}+j(i)+l\right)}{\Gamma(j(i))} \\
\delta_{(j(i), j(k)-1)} \frac{2^{-l} \Gamma\left(\frac{3}{2}+j(i)+l\right)}{\Gamma(j(i))}
\end{array}\right. \\
& \times\left(\frac{1}{2} \delta_{o(i)}^{o(k)}+\left(1-\delta_{o(i)}^{o(k)}\right) \cdot|o(i)-o(k)|\right)
\end{aligned}
$$

where $|\cdot|$ is the absolute sign. We now denote the operator $M_{x_{1}}^{x_{2}}=\min \left(x_{1}, x_{2}\right)$ for the minimal value of $x_{1}, x_{2}$ and $H_{x}$ the Heaviside step function with $H_{x}=$ 1 iff $x \geq 0$. The last term $\mathbf{U}_{i k}^{\mathrm{III}}$ evaluates to

$$
\begin{aligned}
\mathbf{U}_{i k}^{\mathrm{III}} & =\frac{u_{t}^{3}}{u_{s}^{3}} \delta_{j(k)}^{j(i)} \delta_{l(k)}^{l(i)} \delta_{m(k)}^{m(i)} \frac{2^{-(l+2)} \Gamma(j(i)+l+1 / 2)}{\pi^{2} \Gamma(j)} \times\left(\frac{1}{4}|o(i)-o(k)|+\frac{1}{16} \delta_{o(i)}^{o(k)}+M_{o(i)}^{o(k)}\right. \\
& +\sum_{p=1}^{M_{o(i)}^{o(k)}+1}(o(i)-p)(o(k)-p) H_{M_{o(i)}^{o(k)}-p}+H_{o(i)-1} H_{o(k)-1}(o(i)+o(k)-2 \\
& \left.\left.+\sum_{p=0}^{M_{o(i)-1}^{o(k)-2}} p+\sum_{p=0}^{M_{o(i)-2}^{o(k)-1}} p+M_{o(i)-1}^{o(k)-1}(|o(i)-o(k)|-1) H_{(|o(i)-o(k)|-1)}\right)\right)
\end{aligned}
$$

We finally compute the complete $3 \mathrm{D}+\mathrm{t}$ Laplacian regularization matrix as

$$
\mathbf{U}=\mathbf{U}^{\mathrm{I}}+\mathbf{U}^{\mathrm{II}}+\mathbf{U}^{\mathrm{III}}
$$




\section{References}

1. Y. Assaf, T. Blumenfeld Katzir, Y. Yovel and P. Basser. "AxCaliber: a method for measuring axon diameter distribution from diffusion MRI." Mag Reson Med, 59.6, 1347-1354, (2008).

2. E. Stejskal, "Use of Spin Echoes in a Pulsed Magnetic Field Gradient to Study Anisotropic, Restricted Diffusion and Flow." J Chem Phys 43.10, 3597-3603, (1965).

3. P. Basser, J. Mattiello and D. LeBihan. "Estimation of the Effective Self-Diffusion Tensor from the NMR Spin Echo." J Mag Reson, Series B 103.3, 247-254, (1994).

4. S. Merlet and R. Deriche. "Continuous Diffusion Signal, EAP and ODF Estimation via Compressive Sensing in Diffusion MRI." Med Im Anal 17.5, 556-572, (2013).

5. E. Özarslan, C. Koay, T. Shepherd, M. Komlosh, M. İrfanoğlu, C. Pierpaoli and P. Basser. "Mean apparent propagator (map) mri: a novel diffusion imaging method for mapping tissue microstructure." NeuroImage 78, 16-32, (2013).

6. D. Barazanyand, D. Jones and Y. Assaf. "Axcaliber 3D" ISMRM 2011

7. A. Bar-Shir, L. Avram, E. Özarslan, P. Basser and Y. Cohen. "The effect of the diffusion time and pulse gradient duration ratio on the diffraction pattern and the structural information estimated from q space diffusion MR: Experiments and simulations." J Mag Reson 194.2, 230-236, (2008).

8. E. Özarslan, E. Shemesh, N. Koay, C. Cohen and P. Basser. "Nuclear magnetic resonance characterization of general compartment size distributions." New journal of physics 13.1, 015010, (2011).

9. G. Sanguinetti and R. Deriche. "Mapping Average Axon Diameters Under Long Diffusion Time", ISBI (2014).

10. P. Callaghan. "Pulsed-gradient spin-echo NMR for planar, cylindrical, and spherical pores under conditions of wall relaxation." JMR, Series A, 113(1), 53-59, (1995).

11. Sparse Reconstruction Challenge for Diffusion. http://projects.iq.harvard. edu/sparcdmri/challenge

12. M. Descoteaux, E. Angelino, S. Fitzgibbons and R. Deriche. "Regularized, fast, and robust analytical Qball imaging". MRM, 58(3), 497-510, (2007).

13. P. Craven and G. Wahba. "Smoothing Noisy Data with Spline Functions." NUMER MATH 31.4, 377-403, (1978).

14. P. Cook, Y. Bai, S. Nedjati-Gilani, K. Seunarine, M. Hall, G. Parker and D. Alexander (2006, May). Camino: open-source diffusion-MRI reconstruction and processing. ISMRM 14th scientific meeting (Vol. 2759).

15. D. Alexander, P. Hubbard, M. Hall, E. Moore, M. Ptito, G. Parker and T. Dyrby. "Orientationally invariant indices of axon diameter and density from diffusion MRI." Neuroimage 52.4, 1374-1389, (2010).

16. Y. Assaf and P. Basser. "Composite hindered and restricted model of diffusion (CHARMED) MR imaging of the human brain. Neuroimage, 27(1), 48-58, (2005).

17. Y. Assaf, R. Freidlin, G. Rohde and P. Basser. "New modeling and experimental framework to characterize hindered and restricted water diffusion in brain white matter." MRM 52.5, 965-978, (2004).

18. E. Caruyer, C. Lenglet, G. Sapiro and R. Deriche. "Design of multishell sampling schemes with uniform coverage in diffusion MRI". MRM, 69(6), 1534-1540, (2013).

19. T. Dyrby, W. Baaré, D. Alexander, J. Jelsing, E. Garde and L. Søgaard, "An ex vivo imaging pipeline for producing high quality and high resolution diffusion weighted imaging datasets". HBM, 32(4), 544-563, (2011).

20. Garyfallidis E, Brett M, Amirbekian B, Rokem A, van der Walt S, Descoteaux M, Nimmo-Smith I and Dipy Contributors (2014). Dipy, a library for the analysis of diffusion MRI data. Frontiers in Neuroinformatics, vol.8, no.8. 